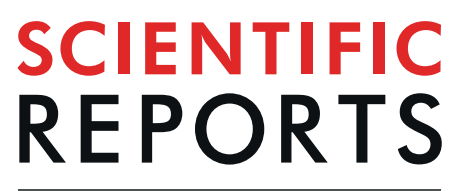

natureresearch

\title{
AutoCLEM: An Automated Workflow for Correlative Live-Cell Fluorescence Microscopy and Cryo- Electron Tomography
}

\author{
Xiaofeng Fu ${ }^{1}$, Jiying Ning ${ }^{1}$, Zhou Zhong ${ }^{2}$, Zandrea Ambrose ${ }^{2}$, Simon Charles Watkins ${ }^{3}$ \& \\ Peijun Zhang $\mathbb{1}^{1,4,5^{*}}$
}

Correlative light and electron microscopy (CLEM) combines the strengths of both light and electron imaging modalities and enables linking of biological spatiotemporal information from live-cell fluorescence light microscopy ( $\mathrm{fLM}$ ) to high-resolution cellular ultra-structures from cryo-electron microscopy and tomography (cryoEM/ET). This has been previously achieved by using fLM signals to localize the regions of interest under cryogenic conditions. The correlation process, however, is often tedious and time-consuming with low throughput and limited accuracy, because multiple correlation steps at different length scales are largely carried out manually. Here, we present an experimental workflow, AutoCLEM, which overcomes the existing limitations and improves the performance and throughput of CLEM methods, and associated software. The AutoCLEM system encompasses a highspeed confocal live-cell imaging module to acquire an automated fLM grid atlas that is linked to the cryoEM grid atlas, followed by cryofLM imaging after freezing. The fLM coordinates of the targeted areas are automatically converted to cryoEM/ET and refined using fluorescent fiducial beads. This AutoCLEM workflow significantly accelerates the correlation efficiency between live-cell fluorescence imaging and cryoEM/ET structural analysis, as demonstrated by visualizing human immunodeficiency virus type 1 (HIV-1) interacting with host cells.

Cryogenic correlative light and electron microscopy (cryoCLEM) has become a valuable tool for investigating the high-resolution ultra-structures of specific biological entities and events in situ in their near-native states, owing to the combined advantages of both imaging modalities. On one hand, fluorescence light microscopy (fLM) enables spatial localization and analyses of dynamic properties of molecules in cellular processes. Particularly, proteins, macromolecular complexes and organelles that have been labeled with fluorescence probes can be localized, tracked, and distinguished from the crowded content of the cell, however, with limited spatial resolution (dozens of nanometers at best in super-resolution mode ${ }^{1}$ ). On the other hand, cryo-electron tomography (cryoET) has emerged as a powerful technique for visualizing three-dimensional (3D) cellular structures at high resolution in their near-native state and natural environment ${ }^{2}$. With the recent development of subtomogram averaging techniques, cryoET is now able to generate detailed views of cellular components at sub-nanometer resolutions ${ }^{3-6}$. However, the information obtained from cryoET is limited to 3D snapshots of a biological event at specific time points. To achieve a time-lapse panorama and capture meaningful scenes during biological processes, such as virus entry, budding or cell signaling, combined live-cell imaging and high-resolution cryoET with subtomogram averaging using cryoCLEM, is especially desirable ${ }^{7,8}$.

One challenging aspect of cryoCLEM is to image fluorescence signals at cryogenic temperature in order to keep the sample vitrified. For this purpose, several groups have developed cryo-light microscopy stages ${ }^{7,9-14}$, cryofLM probes $^{15,16}$ and methods ${ }^{17-21}$ for cryofLM imaging. Recently cryofLM devices have become available

\footnotetext{
${ }^{1}$ Department of Structural Biology, University of Pittsburgh School of Medicine, Pittsburgh, PA, 15260, USA. ${ }^{2}$ Department of Microbiology and Molecular Genetics, University of Pittsburgh School of Medicine, Pittsburgh, PA, 15260, USA. ${ }^{3}$ Department of Cell Biology and Physiology, University of Pittsburgh School of Medicine, Pittsburgh, PA, 15260, USA. " Division of Structural Biology, Wellcome Trust Centre for Human Genetics, University of Oxford, Oxford, OX3 7BN, UK. ${ }^{5}$ Electron Bio-Imaging Centre, Diamond Light Sources, Harwell Science and Innovation Campus, Didcot, OX11 0DE, UK. *email: peijun@strubi.ox.ac.uk
} 
from several commercial sources (FEI Corrsight, Leica EM CryoCLEM ${ }^{22}$, Zeiss CryoAiryscan and Linkam). Such systems have been successfully applied to image neurons, viruses, mitochondria and many other cellular components $s^{8,12,14,19,23-28}$. To improve the accuracy and efficiency of cryoCLEM, various probes (visible in both fLM and EM) have been employed to guide the correlation step; these include toner particles ${ }^{29}$, FluoroNanogold $(\mathrm{FNG})^{30}$, quantum dots $(\mathrm{QDs})^{31}, 3,3^{\prime}$-diaminobenzidine (DAB after photoconversion) ${ }^{32}$, fluorescent dye beads $s^{13,21,22,33}$, and cathodoluminescence pointers $^{34}$. Further, on the EM side, automation software, such as Leginon ${ }^{35}$, serialEM ${ }^{36}$ and ThermoFisher/FEI EPU/TOMO, have allowed users to generate grid atlas maps and to import fLM maps for alignment and registration.

However, the entire process of cryoCLEM is (i) tedious and time-consuming due to difficulties in localizing the corresponding region of interest (ROI) under $\operatorname{cryoEM}^{19,20}$, (ii) inefficient with low throughput due to multiple steps of manual operation and correlation, and (iii) difficult due to low signals in cryoEM that make direct correlation a challenge. To simplify the cryoCLEM procedure and to make it more robust and accurate, we developed AutoCLEM, a workflow employing fluorescent fiducial beads for automated alignment of correlative images in three sequential steps, from low-resolution fLM to low and medium magnification cryoEM, and to high resolution cryoET. We applied it to examine the early stages of infection by human immunodeficiency virus type 1 (HIV-1) and demonstrate a greatly reduced investment of time for correlation processes (10 fold) as well as improved accuracy ( 5 fold).

\section{Results}

Overview of autoCLEM workflow. The conventional cryoCLEM experiment usually involves four main steps: I) imaging fixed or live cells with fLM, II) plunge-freezing of cells on EM grids, III) cryofLM imaging, and IV) cryoEM/ET data collection (Fig. 1A). The key to successful cryoCLEM is a reliable and effective correlation between fLM in step III and cryoEM/ET in step IV. As illustrated in Fig. 1B, the AutoCLEM workflow comprises three levels of correlation between fLM/cryofLM and cryoEM/ET, to automatically localize targets of interest (e.g. HIV-1 virus particles) for cryoEM/ET structural analysis. The experimental setup for cell culture on EM grids and subsequent viral infection is shown in Supplementary Fig. S1. The $200 \mathrm{~nm}$ FluoSphere beads were pre-coated on the backside of the EM grids to avoid their endocytosis by cells (Supplementary Movie S2, Supplementary Fig. S3) and to minimize bead movement or loss during plunge freezing.

The first step of correlation is at the level of grid-square, indicated as 'square' correlation (Fig. 1Bi-iii). It is not trivial to directly align these two overview maps from fLM (Fig. 1Bi) and cryoEM (Fig. 1Bii,iii), because the cryoEM map clearly has different intensity characteristics from fLM map, including the presence of intensity gradients with totally dark regions due to the thick ice. Nevertheless, the common features such as the grid bars and characters on the 'finder' grid aid alignment of the two maps. After rescaling and thresholding the intensity of the maps, the two overview maps can be aligned automatically using a homemade script in AutoCLEM software (Supplementary material) by cross-correlation. This alignment is done with the IMOD command 'xfalign' where the best match is found at the highest cross-correlation value by varying rotation angle and slightly refining magnification. The coordinates for the center of interested grid-squares from fLM are mapped onto the corresponding squares in cryoEM (Fig. 1Bi,iii, green dots). This 'square' correlation can tolerate certain grid defects or deformations, which are common to cryoEM grids since they are easily damaged during freezing, loading or transfer. In fact, using a cryoCLEM sample stage that accepts an autogrid ${ }^{22}$ or a polara cartridge ${ }^{7}$ minimizes direct manual handling of EM grids, thus, greatly reduces the artifact.

The next step of correlation is at the fluorescent bead level, indicated as 'bead' correlation (Fig. 1Biv-vi). This step generates a transformation matrix between the fLM image at $100 \times$ magnification (Fig. 1Biv) and the cryoEM grid-square map at $4,700 \times$ magnification (Fig. $1 \mathrm{Bv}$ ) using $200 \mathrm{~nm}$ fluorescent beads. The grid-square map (Fig. $1 \mathrm{Bv}$ ) is a $12 \times 12$ montage of cryoEM images recorded at $4,700 \times$, a magnification at which the beads are visible and easily distinguished from ice or other contaminants (Fig. 1Bv-vi). Typically, at least 4-5 beads close to the cell periphery are manually or automatically picked from the fLM image (Fig. 1Biv) and from the cryoEM grid-square map (Fig. 1Bv). A Matlab script (Supplementary material) is developed to compute the transformation matrix (Fig. 1Bvi), which allows fitting of parameters including magnification, rotation, translation, and distortion between these two maps based on the selected beads.

The last step of correlation, indicated as "virus" correlation in Fig. 1, is to convert the fLM-extracted ROI coordinates (in this case, virus particles) into cryoEM stage positions by applying the transformation matrix generated from step 2. A high magnification cryoET tilt series can then be collected automatically at the computed position for the ROI (Fig. 1Bviii), considering a calibrated microscope alignment between medium $(4,700 \times)$ and high $(39,000 \times)$ magnifications. These three successive steps are entirely automatic in most cases, with the exception when fLM signals are complicated or overcrowded calling for the need to manually pick viruses and beads. We describe detailed autoCLEM workflow and results in the following sessions using HIV-1 infection as an example.

Automated fLM grid-scan for target selection (Step 1). In previously reported CLEM methods, the correlation process required manually recording the grid-squares selected for confocal fLM imaging and later finding them in cryoEM. This could be difficult when 1) no recognizable or usable feature on the 'finder' EM grid is present to help correlate grid-squares; or 2) the grid is flipped during loading for cryoEM. To get around this manual step, we utilized a new feature of the fLM software NIS-Elements, 'scan large map', to automatically generate an overview at $20 \times$ magnification, Differential Interference Contrast ${ }^{37}$ (DIC) map $(5 \times 5$ tiles are shown) of the entire EM grid in less than 5 minutes with auto-focusing (Fig. 2A). Furthermore, the live-cell fLM overview map provides high quality images that allow for examination of cells and selection of regions for high magnification $(100 \times)$ imaging. For example, the user can simply use this map to select the most appropriate grid-squares or regions over the entire grid within which only 1-2 cells are located (too many crowded cells will produce too thick ice during plunge freezing), and where the cells are mostly spread out, between two mitosis phases. This 

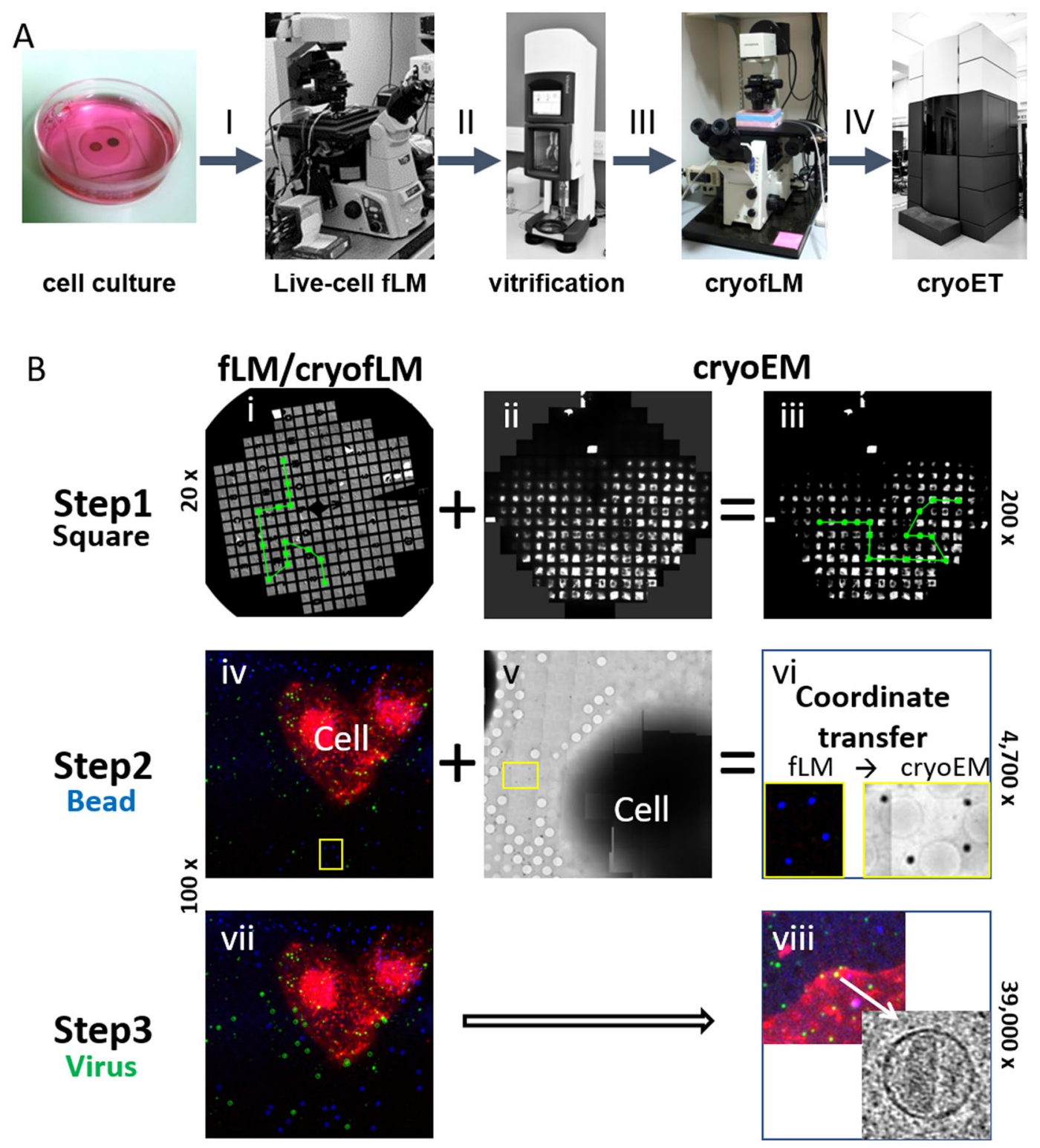

Figure 1. Workflow for AutoCLEM. (A) Four main steps in cryo-correlative light and electron microscopy (cryoCLEM). (B) Schematic of AutoCLEM. Three sequential steps of automated correlation are illustrated. Step1: Low magnification fLM (i, 20×) and cryoEM (ii-iii, 200×) for grid overview, showing that 11 target squares (green dots) are automatically correlated. Step 2: High magnification fLM image $(100 \times)$ to identify areas of interest (iv, yellow box, four blue beads) and the corresponding cryoEM image recorded at a medium magnification $(4,700 \times)$ ( $\mathrm{v}$-vii, four beads). Based on beads, a coordinate transfer matrix is generated between fLM (iv) and cryoEM (v). Step 3: High magnification $(39,000 \times)$ for high resolution cryoET. Particles of interest (vii, green) are localized for cryoET data collection (viii) by applying the transform obtained from step 2 . Beads, HIV-1 particles and cells are in blue, green and red, respectively.

fLM overview map is correlated automatically to the cryoEM grid scan overview map (Supplementary Fig. S2) to facilitate selection of targeted grid-squares for the next level of correlation for higher precision.

The selected grid-squares, such as those indicated in Fig. 1B (green dots) and Fig. 2A,B (labeled as c-e), are then further inspected at higher magnification $(100 \times)$ with automatic recording of DIC and several fluorescence channels, including cell tracker (red) and fluorescent fiducial beads (blue). The cell tracker signal is particularly useful for determining the cell boundary since, for cryoEM, we are interested in the very thin cell periphery, which is almost invisible in the DIC mode, but clearly revealed with cell tracker (Fig. 2C-E). Meanwhile, the fluorescent bead distribution is also clearly exhibited (Fig. 2C-E, blue). Criteria for selecting good grid-squares include 1) the presence of only $1 \sim 2$ cells with the cell periphery located close to the center of the square, and 2) the presence of at least $4 \sim 5$ beads around the cell periphery of interest. 

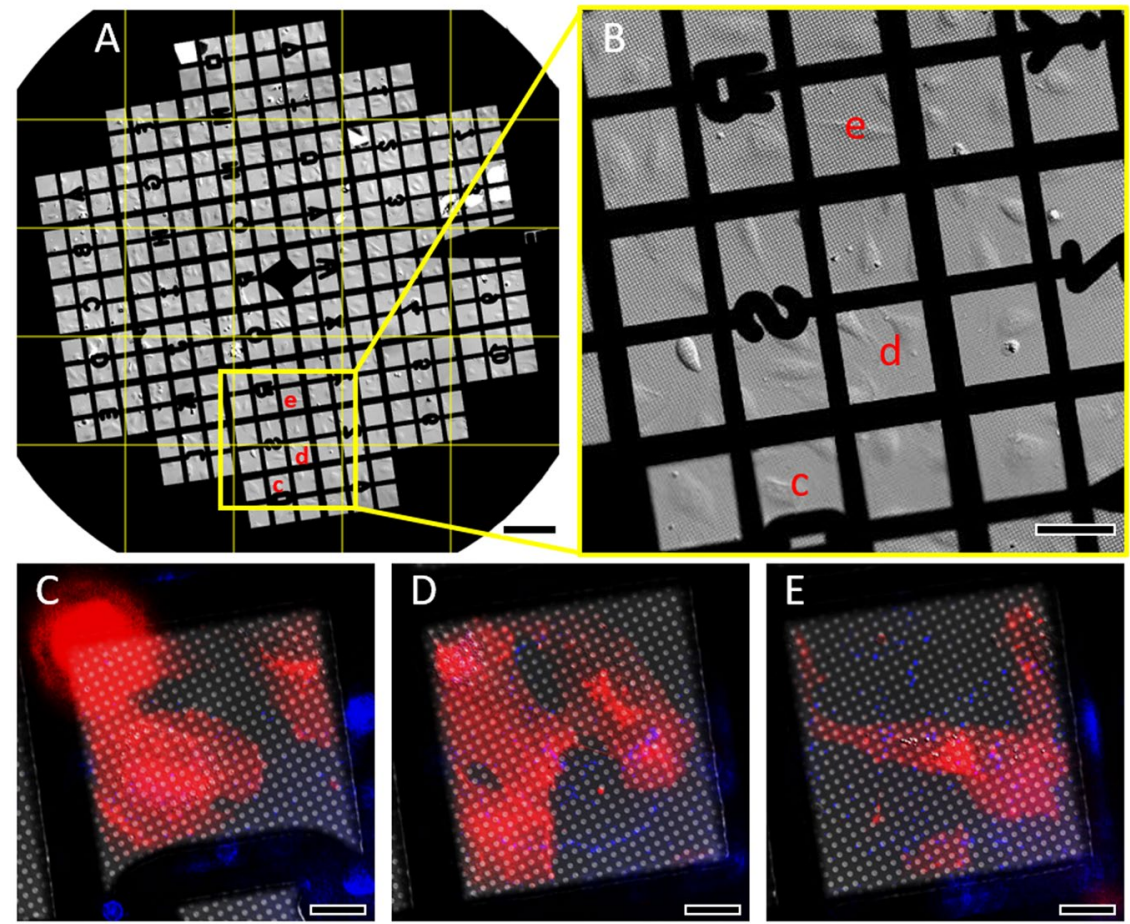

Figure 2. Automatic grid scan with fLM and target square selection. (A) An EM grid was automatically scanned for a $5 \times 5$ array with fLM at 20x DIC mode, stitched for grid montage. (B) An area (yellow box in A) is zoomed in to clearly view cell concentration, morphology and distribution in each grid square, to allow preselection of appropriate squares. These pre-selected squares ('c-e' in B) are then further inspected by automatic collection in three channels (DIC, cells (red) and beads (blue)) with fLM at 100x (C-E). The cell membrane dye (red) shows much clearer cell boundary than DIC channel (C\&D) because the HUVEC cell periphery is very thin. The blue signal from beads help to guide selection of regions with proper bead distribution. The scale bar is $200 \mu \mathrm{m}$ in $\mathrm{A}, 100 \mu \mathrm{m}$ in $\mathrm{B}$, and $20 \mu \mathrm{m}$ in $(\mathbf{C}-\mathbf{E})$, separately.

Automated confocal live-cell microscopy (Step 2). Live-cell imaging with fLM is a key step in capturing cellular processes at a stage of interest, for example, during the early stages of HIV-1 infection. In this case, high-speed confocal live-cell imaging at pre-selected grid squares was performed during the first 40-60 minutes after the addition of HIV-1 virus particles (Fig. 3, green). These virus particles carry a mutation E45A on the capsid protein (CA), which causes the capsid to be hyper-stable ${ }^{38,39}$ making it easier to capture intact capsids in imaging studies compared to wild-type virus particles ${ }^{7}$. To image HIV-1 and host cell interactions by cryoET, we targeted thin $(<300 \mathrm{~nm})$ periphery regions of the cell. High-speed time-lapse confocal fLM images were automatically recorded, with Z-sections from the fiducial beads located on the back-side carbon film to the top surface of the cell (Fig. 3, Supplementary Movie S1). Both virus particles (green) and cells (red) were imaged simultaneously during the entire time-lapse confocal live-cell imaging period, while the image of beads (blue) was collected only once, at the end of confocal live-cell imaging (Fig. 3C). From the live-cell confocal series, we were able to track HIV-1 particles in 3D (Supplementary Movie S1), revealing fast virus particle movements over the 40 minute post-infection when particles were associated (overlapped) with the cell (Fig. 3B); however, a majority of virus particles were relatively stationary and located away from the cell (Fig. 3B). Focusing on the area marked with a cyan box in Fig. 3B, one virus particle displayed rapid movement in a curvilinear path in the cell periphery (Fig. 3D1-11, arrows), consistent with previous observations that particles traffic along microtubules within the cytoplasm ${ }^{8,40}$. Within the same area, two other virus particles displayed much less motion (Fig. 3D1-11, arrowheads).

Automated fiducial correlation and virus particle localization (Step 2-3). Confocal live-cell images of virus particles (green), fluorescent beads (blue) and cells (red) were recorded simultaneously at the end of time-lapse series, and these were projected in 2D, as visualized in the overlay image (Fig. 4A). The information in the $Z$ direction was omitted in this case but could be very useful when cryoCLEM processing includes cryo-focused ion beam (cryoFIB) milling in a scanning electron microscope. From the 2D images, four blue beads were manually selected using IMOD software ${ }^{41}$ (Fig. 4C, blue box). Once the corresponding cryoEM grid-square map was acquired (Fig. 4B), the same beads identified in the fLM projection image (Fig. 4A) were identified in the cryoEM map, and their corresponding coordinates automatically saved in a SerialEM navigation file (Fig. 4C, blue box). A Matlab script was developed (Supplementary material) to calculate the transformation matrix between fLM and cryoEM map based on the bead coordinates from the two microscope systems. The core part of this script is called 'estimateGeometricTransfrom'. It can calculate the scaling, translation, rotation and 

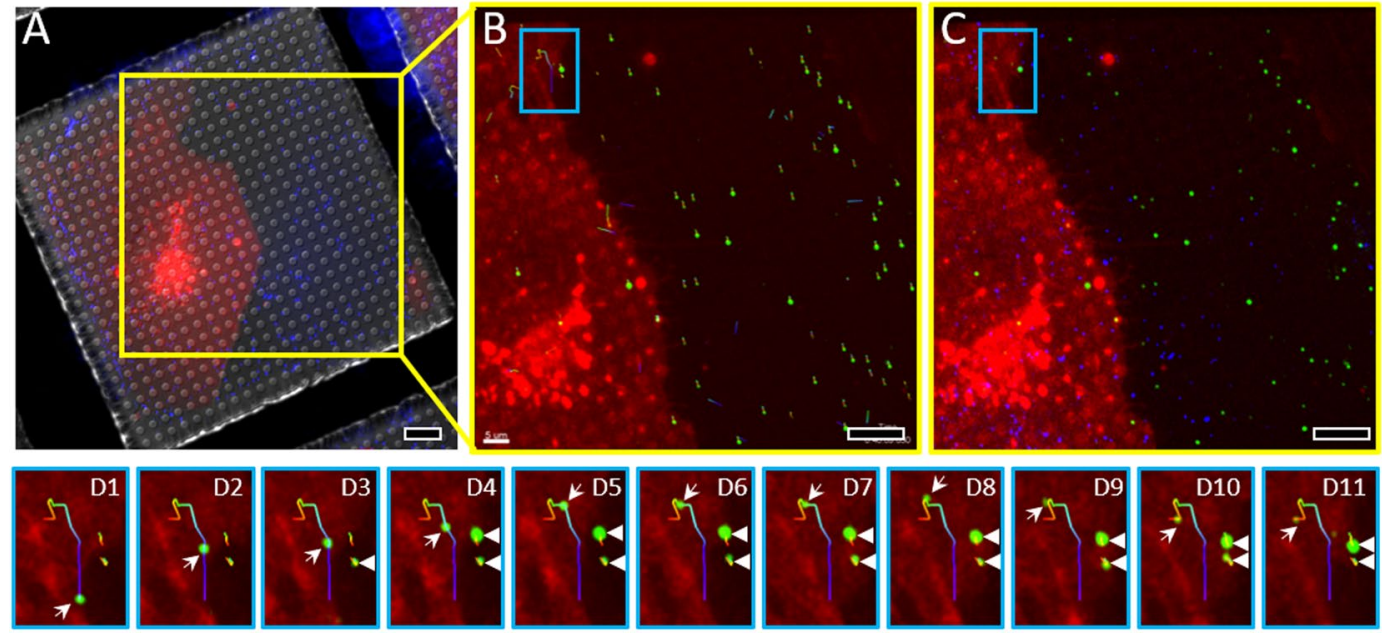

Figure 3. Automated confocal live-cell microscopy. (A) A typical grid square with overlaid signals from three channels, DIC, red (cells) and blue (beads). (B,C) The last time point of confocal image (yellow box area in panel A, shown overlaid signals from red (cells), green (HIV-1 viruses) (B) and blue (beads) (C). The green viruses are tracked in 3D from time-lapse confocal live-cell images, shown in B (thin line tracks). (D1-11) Enlarged views of a time-lapse series of an infecting virus (in cyan box area in B,C) from time 0 to 40 minutes with an interval of 4 minutes. The scale bars are $10 \mu \mathrm{m}$ in $(\mathbf{A}-\mathbf{C})$.

distortion between fLM and cryoEM images. The script works quite well even when there appears to be some grid bending, which frequently happens during freezing or grid handling. The initial coarse positioning error, measured at a low cryoEM magnification $(4,700 \times)$, is about $0.74 \mu \mathrm{m}(\mathrm{n}=33)$, which is further reduced to $0.13 \mu \mathrm{m}$ $(\mathrm{n}=17)$, measured at high magnification for cryoET $(39,000 \times)$.

After generating the coordinate transfer matrix (Fig. 4C, yellow box), we selected eight extracellular virus particles, as these were expected to have been stationary before freezing (Fig. 4A, green circles). The virus coordinates were extracted from the fLM 2D projected image and converted to cryoEM stage positions (Fig. 4C, green boxes). The SerialEM navigator file was then updated with the calculated virus coordinates in EM, followed by automated tomography data acquisition through all selected virus particles with SerialEM. The error for positioning virus particles was analyzed to be $\sim 100 \mathrm{~nm}(\mathrm{n}=13)$, which is well within the tolerant range for cryoEM imaging at the high magnification, typically with an imaging frame of $\sim 1.0 \mu \mathrm{m} \times 1.0 \mu \mathrm{m}$ for a pixel size of $2.8 \AA$. The tomographic slices from the eight selected virus particles (Fig. 4A, green circles) are presented in Fig. 4D-K. These virus particles display a variety of morphologies, including defective immature particles (D), immature particles (E), defective mature particles (F), maturation intermediates (G-I) and mature particles with clear conical-shaped cores $(\mathrm{J}-\mathrm{K})^{42}$. All viruses show clear envelope spikes as they are located outside of cells.

Auto-cryoCLEM of HIV-1 infection (Step 3). The initial AutoCLEM work was performed with confocal live-cell fLM at room temperature. This works especially well for static objects that do not move significantly during the 5-10 minutes gap between the last fLM image and plunge-freezing. However, in cases when the objects of interest are dynamic, for example the infecting virus particle shown in Fig. 3D, a direct correlation between cryofLM and cryoEM images after plunge-freezing is necessary. We tested AutoCLEM performance using the cryofLM setup that we reported previously ${ }^{7}$, by first collecting fLM images at the end of the confocal live-cell series and then cryofLM images after plunge-freezing. As shown in Fig. 5, both 2D projected confocal live-cell fLM (Fig. 5A) and cryofLM (Fig. 5B) images, before and after freezing respectively, displayed a similar pattern for the fluorescent beads, as expected (blue circles). Using the AutoCLEM script, the location of a virus particle identified in the cryofLM image was mapped onto the cryoEM image for tomography data collection (Fig. 5C, green). A mature HIV-1 virus bound to the cell surface and prior to fusion was captured and imaged. The slice views of the corresponding tomogram show ribosome molecules inside the cell (Fig. 5D,E), followed by the enveloped virus particles bound to the cell surface below (Fig. 5E-G, arrow), alongside an exosome (Fig. 5F,G, arrowhead).

\section{Discussion}

The EM grids used in this protocol are Au or Ni finder Quantifoil grids, illustrated in Supplementary Fig. S1. The finder landmarks can be easily identified in both fLM and low magnification cryoEM and are necessary for three reasons: 1) the landmarks help users to identify the sample side before plunge freezing; 2) the landmarks help to identify whether the grid is flipped during cryoEM loading; 3) the landmarks help manual grid-square correlation in cases when the ice on the frozen grid is too thick. The use of nickel grids aids autofocus functionality in light microscopy during grid scan, but with a caveat of inducing image/beam shift during tomography data collection.

We have tested different FluoSphere dye beads for their utility as fiducial markers. The $100 \mathrm{~nm}$ bead is bright enough to be visible at $100 \times$ live-cell confocal $\mathrm{fLM}$ imaging and at $40 \times$ cryofLM but are difficult to distinguish at medium magnification cryoEM, especially around the cell periphery where the ice is relatively thick $(\sim 300 \mathrm{~nm})$. The $1 \mu \mathrm{m}$ bead also was not suitable, as beads of this size are easily blotted away during plunge freezing, whereas $100 \mathrm{~nm}$ and $200 \mathrm{~nm}$ sized beads remain on the EM grid. In addition, precisely determining the positions of the 

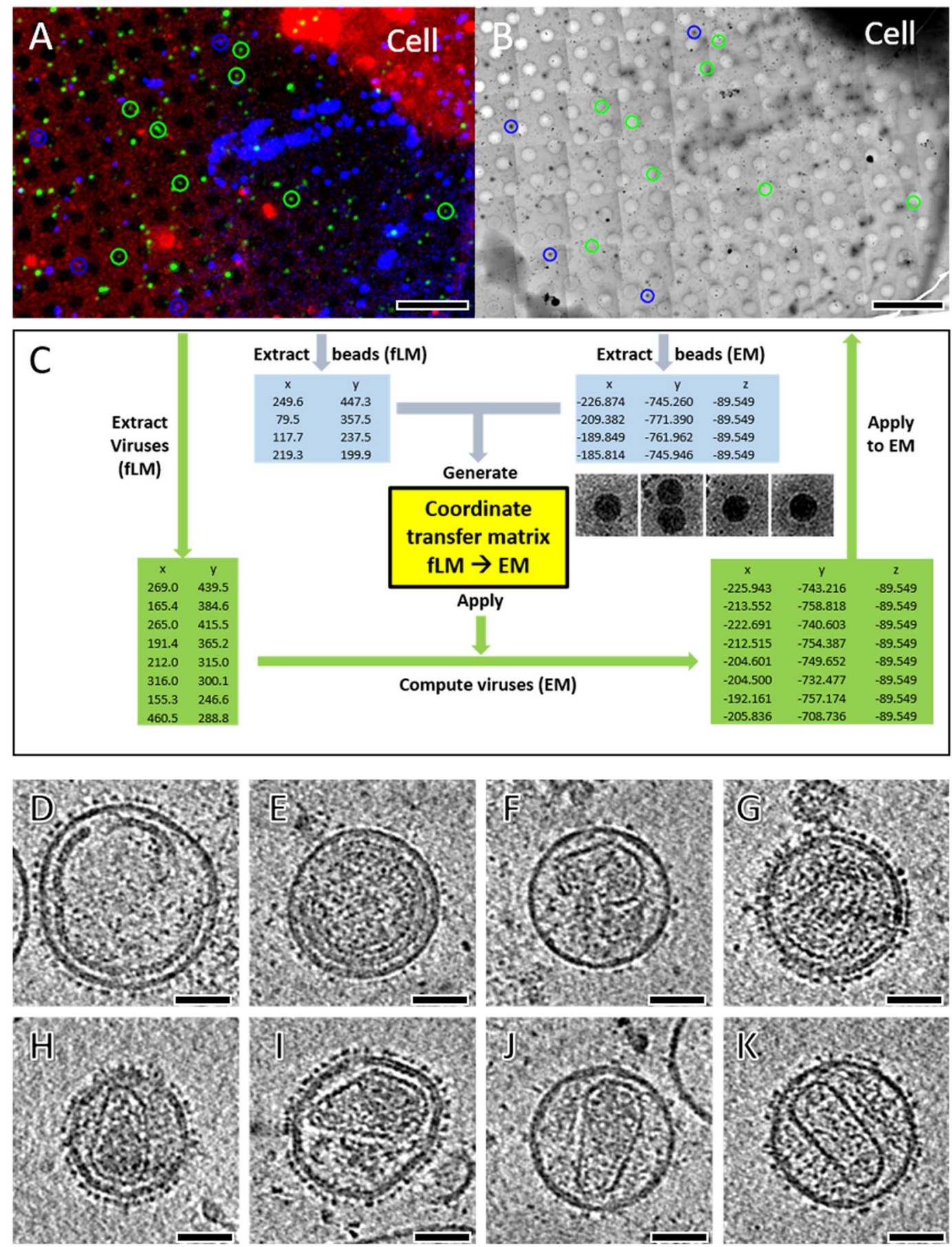

Figure 4. Automated (or semi-automated) correlation of beads and localization of HIV-1 particles. (A) The last confocal fLM image for a selected square with red (cells), green (viruses) and blue (beads) signals. (B) The corresponding cryoEM image at medium magnification $(4,700 \times)$ showing bead positions. Four individual beads or bead aggregates (blue circles) were selected for correlation. (C) The illustration of workflow for bead correlation and viral localization. The coordinates of four beads (in blue) both from fLM and cryoEM images are extracted, from which the coordinate transfer matrix (in yellow) is generated with the consideration of scaling, rotation, translation and distortion. Applying the transfer matrix to virus coordinates (in green), extracted from fLM image, enables computing their coordinates (in green) in cryoEM. (D-K) Central slices of reconstructed tomograms from 8 correlated virus particles in panel $(\mathbf{A}, \mathbf{B})$ display various morphologies: defective immature particle $(\mathbf{D})$, immature particle $(\mathbf{E})$, defective mature particle $(\mathbf{F})$, maturation intermediates $(\mathbf{G}-\mathbf{I})$ and mature particles $(\mathbf{J}, \mathbf{K})$. The scale bars are $10 \mu \mathrm{m}$ in A-B and $50 \mathrm{~nm}$ in $(\mathbf{D}-\mathbf{K})$, respectively. 

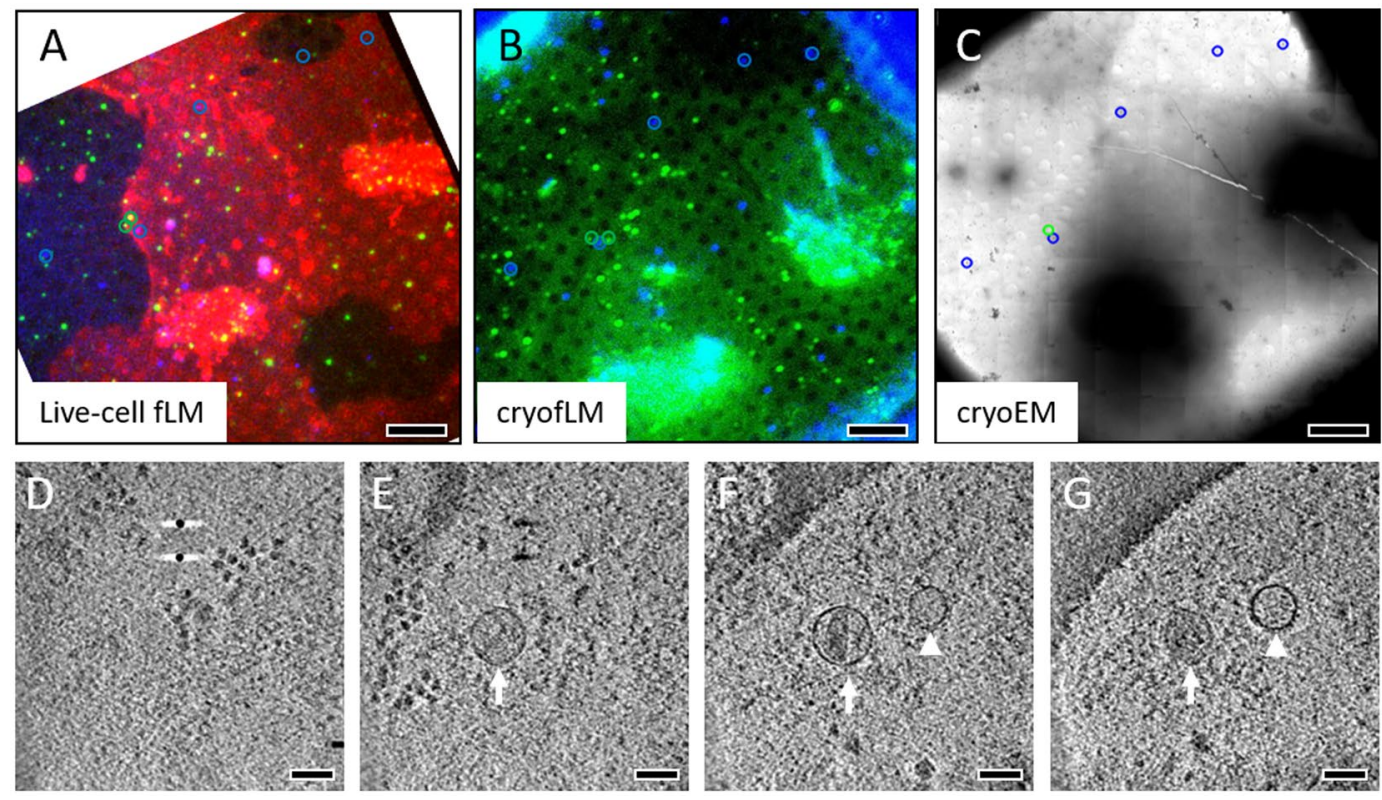

Figure 5. Tomographic imaging of HIV-1 particles bound to a cell using cryo-AutoCLEM. (A-C) The grid square images are displayed for confocal fLM at the last time point (A), cryofLM after freezing (B) and the cryoEM medium magnification scan $(\mathbf{C})$. The beads were pre-coated on the EM grid and show a fixed pattern (blue circles). The virus particles (green circles) moved during a time gap of few minutes, between live-cell fLM and plunge freezing. The virus coordinate in cryoEM $(\mathbf{C})$ was computed from the coordinate extracted from cryofLM image (B) using the transfer matrix generated from the bead coordinates (blue circles). (D-G) Four successive slice views of the tomogram acquired from the area containing correlated virus particle (arrow), along with an exosome (arrowhead). The scale bar is $10 \mu \mathrm{m}$ in $(\mathbf{A}-\mathbf{C})$, and $100 \mathrm{~nm}$ in $(\mathbf{D}-\mathbf{G})$, respectively.

$1 \mu \mathrm{m}$ beads in cryoEM is a challenge, as they often retain a significant amount of liquid during freezing, which obscures the shape of beads in cryoEM. We found that $200 \mathrm{~nm}$ beads work best as fiducial markers compared to either smaller or larger beads. We have also discovered that the beads must be pre-coated on the back side (non-sample side) of the EM grid, or added instantly before plunge freezing. If added to the sample side, the beads are taken up by the cells through endocytosis, as shown in Supplementary Fig. S3 and Supplementary Movie S2.

Previously reported CLEM methods include multiple steps of correlation, beginning from low magnification and employing either internal grid features (grid bar and edge or characters) or external particles (toner particles, random contamination) for manual correlation of grid-squares or areas of interest, followed by medium or high magnification correlation using immunogold, Quantum Dots, dye beads, holes on Quantifoil grid carbon, or even intrinsic cellular features as fiducial markers ${ }^{13,21,22,29-34}$. Since most of the correlations were performed manually, the procedures were very time-consuming and tedious. The autoCLEM workflow presented here comprises a near-fully automatic three-step correlation procedure; only the picking of fiducial beads in fLM image and cryoEM grid-square maps for calculating the transformation matrix is manual, and this takes less than 5 minutes. In principle, this manual aspect of the procedure can also be automated in AutoCLEM, but in practice, the variability of fluorescence signals among beads, the amount of ice contamination and variable ice thickness make this challenging. All together the grid-square correlation process (step 1) can be shortened to 10 minutes from 1 hour typically required in a manual effort, and the bead correlation and viral localization steps (steps 2-3) can be shortened to 10 minutes per square from previous 2 hours per square (Supplementary Table S1). Most importantly, the accuracy of target positioning is five times better $(\sim 500 \mathrm{~nm}$ to $\sim 100 \mathrm{~nm})$, which could be further improved when super-resolution cryoCLEM is performed ${ }^{15}$. The current challenge for a completely automated process is the frequent mismatch of bead patterns between fLM/cryofLM and cryoEM, due to missing fiducial beads in either system. We are developing methods to improve the detection reliability and correlation accuracy towards a fully automated AutoCLEM, as demonstrated in Supplementary Fig. S4, in which a correlation of beads was established even though the detection of beads was incomplete in both systems. This resulted in automatic localization of virus particles (Fig. S4F-G, pink circles) in cryo grid-square map.

\section{Methods}

Production of VSV-G pseudo-typed HIV-1 particles. As previously described ${ }^{7}$ E45A HIV-1 was produced by transfecting 293T cells with three plasmids: pL-VSV-G (a kind gift from M. Emerman), peGFPC3-Vpr (a kind gift from T. Hope), and a proviral plasmid, pNLdE-luc, encoding HIV-1 $1_{\mathrm{NL4}-3}$ with deletions in env and with the luciferase gene in place of $n e e^{43}$. Transfections were performed in 293T cells with lipofectamine 2000 (Invitrogen, Carlsbad, CA) in the cell medium DMEM (10\% FBS Pen/Strep/Glu) (Gibco). Virus infectivity was determined on GHOST-R3/X4/R5 cells and capsid levels were measured by p24 ELISA (Zeptometrix, Buffalo, NY). 
Cell culture on cryoEM grids. Au or Ni R2/2 Quantifoil 'finder' EM grids (Quantifoil Micro Tools GmbH, Jena, Germany) were glow-discharged on the back-side of the grid at $20 \mathrm{~mA}$ for 20 seconds, coated with $1 \mu \mathrm{l}$ 1000x diluted FluoSphere $200 \mathrm{~nm}$ blue beads (Invitrogen, Eugene, OR), and left to dry. The grids were then disinfected under UV light for 2 hours and treated with $50 \mu \mathrm{g} / \mathrm{ml}$ fibronectin (Sigma-Aldrich, from bovine plasma) on the front-side. Human umbilical vein endothelial cells (HUVEC) were cultured at $37^{\circ} \mathrm{C}$ with $5 \% \mathrm{CO}_{2}$ in $\mathrm{EGM}^{\mathrm{TM}}-2$ BulletKit ${ }^{\mathrm{TM}}$. Cultures at approximately $70-85 \%$ confluence were rinsed with $5 \mathrm{ml}$ room temperature HEPES Buffered Saline (HEPES-BSS) and incubated in Trypsin/EDTA solution ( $0.5 \mathrm{ml}$ Trypsin/EDTA for $60 \mathrm{~mm}$ culture dishes). After about $90 \%$ of the cells were rounded, the cells were diluted in cell medium, to a density of $0.8-1 \times 10^{5} \mathrm{cells} / \mathrm{ml}$, and plated $(2 \mathrm{ml} / \mathrm{dish})$ onto disinfected grids placed in glass-bottom culture dishes (MatTek Corporation, Ashland, MA) with the cell side up. Each dish was incubated overnight.

Live-cell fluorescence imaging. The HUVEC cells were stained for 10 minutes by addition of $1.0 \mu$ of cell tracker, CellMask Deep-Red (ThermoFisher, Waltham, MA, USA.) to the culture medium. After rinsing and replacement of the culture medium, the cells were infected and imaged as follows. During these processes, careful attention should be paid to not disturb the EM grids in the dishes. For fluorescence live-cell imaging, a Nikon Ti widefield microscope, Bruker Optera confocal scan head and Andor Ultra EM CCD camera was used. A Tokai hit environmental chamber was used to maintain temperature and normal $5 \% \mathrm{CO}_{2}$ tissue culture conditions. The system was controlled by NIS Elements software (Nikon instruments, Melville, New York). The EM grid was imaged in glass-bottom dish, which was centered and automatically scanned in DIC channel at $20 \times$ magnification $(20$ $\times 0.75$ NA plan apo lens), in $5 \times 5$ stitched tiles, covering the entire $3.05 \mathrm{~mm}$ EM grid (Fig. $2 \mathrm{~A})$. Grid-squares of interest were selected, and images of these regions were automatically acquired at $100 \times$ magnification $(100 \times$ 1.49 TIRF) in DIC and two fluorescence channels ( $640 \mathrm{~nm}$ red for cell and $405 \mathrm{~nm}$ blue for bead). After the addition of $200 \mu \mathrm{l}$ pseudo-typed E45A HIV-1, time-lapse confocal live-cell imaging was performed immediately on selected areas at $100 \times$ using the high speed confocal head and EMCCD camera. Confocal images were recorded automatically every 5 minutes for 40 minutes, for two fluorescence channels ( $488 \mathrm{~nm}$ for virus and $640 \mathrm{~nm}$ for cell) with triggered sequence illumination and over an $8 \mu \mathrm{m} \mathrm{Z}$ space at $1.0 \mu \mathrm{m}$ spacing. After the time-lapse confocal imaging was finished, a final set of confocal images were collected with an additional fluorescence chan$\mathrm{nel}$ at $405 \mathrm{~nm}$ (for fluorescent beads), along with the two previous fluorescence channels ( $488 \mathrm{~nm}$ for virus and $640 \mathrm{~nm}$ for cell). 3D reconstruction and virus particle tracking were performed using Imaris (Bitplane, Zurich, Switzerland).

Cryo-electron microscopy and tomography. Immediately after fluorescence confocal imaging, $4 \mu \mathrm{l}$ of $15 \mathrm{~nm}$ gold beads were applied to the EM grid, which was subsequently blotted and plunge frozen using an FEI Vitrobot (FEI, Hillsboro, OR) at $\sim 100 \%$ humidity. The frozen grids were clipped into a Polara cartridge and imaged with a home-built cryo-sample stage to record cryo-fluorescence images, as described previously ${ }^{7}$.

The frozen grids were loaded to Polara G3 microscope (FEI Corp., OR.), equipped with a Gatan US4000 4k $\times 4 \mathrm{k}$ Ultrascan CCD (Gatan, Inc., Warrendale, PA) and a Falcon II direct detector (FEI Corp., OR.) camera, for cryoEM analysis and cryoET data collection. A cryoEM overview map (atlas, a low magnification montage, LMM) of the entire grid was recorded using serialEM, with which a $14 \times 14$ tiles LMM overview was recorded at $200 \times$ on the Ultrascan camera. This LMM overview is correlated to the fLM tile images to find the location of the grid-squares of interest.

After the grid-squares of interest were located, a medium magnification montage (MMM) was recorded for each square at $4,700 \times$ on Falcon II camera with $20 \%$ overlap and $300 \mu \mathrm{m}$ defocus. Using the correlative script developed in this study, coordinates of virus particles were determined, and tilt series were collected bidirectionally, with a tilt range of $-60^{\circ}$ to $60^{\circ}$ and $3^{\circ}$ interval, at a nominal magnification of $39,000 \times$, defocus values between $5-8 \mu \mathrm{m}$ and a total dose of $\sim 100$ e/ $\AA^{2}$ on the Falcon II camera. The tilt series were aligned using $15 \mathrm{~nm}$ gold fiducial beads and reconstructed with weighted back-projection algorithm using the IMOD package ${ }^{41}$. The tomograms of viruses were denoised using 3D nonlinear anisotropic diffusion edge enhancing program implemented in IMOD with 6 iterations and a $\kappa$ value of 0.8 to enhance visualization.

\section{Data availability}

The datasets generated during and/or analyzed during the current study are available from the corresponding author on reasonable request. The AutoCLEM Script is freely available from https://github.com/whatanamelikethis/ok/blob/ master/CLEM_align.

Received: 17 June 2019; Accepted: 2 December 2019;

Published online: 16 December 2019

\section{References}

1. Leung, B. O. \& Chou, K. C. Review of Super-Resolution Fluorescence Microscopy for Biology. Applied Spectroscopy 65, 967-980 (2011).

2. Leis, A., Rockel, B., Andrees, L. \& Baumeister, W. Visualizing cells at the nanoscale. Trends Biochem Sci 34, 60-70 (2009).

3. Schur, F. K. et al. An atomic model of HIV-1 capsid-SP1 reveals structures regulating assembly and maturation. Science 353, 506-508 (2016).

4. Nicastro, D. et al. Cryo-electron tomography reveals conserved features of doublet microtubules in flagella. Proc Natl Acad Sci USA 108, E845-853 (2011)

5. Cassidy, C.K. et al. CryoEM and computer simulations reveal a novel kinase conformational switch in bacterial chemotaxis signaling. Elife 4 (2015).

6. Himes, B. A. \& Zhang, P. emClarity: software for high-resolution cryo-electron tomography and subtomogram averaging. Nat Methods 15, 955-961 (2018). 
7. Jun, S. et al. Direct visualization of HIV-1 with correlative live-cell microscopy and cryo-electron tomography. Structure 19, 1573-1581 (2011).

8. Jun, S. et al. Correlative microscopy for 3D structural analysis of dynamic interactions. J Vis Exp (2013).

9. Arnold, J. et al. Site-Specific Cryo-focused Ion Beam Sample Preparation Guided by 3D Correlative Microscopy. Biophys J 110, 860-869 (2016).

10. Li, S. et al. High-vacuum optical platform for cryo-CLEM (HOPE): A new solution for non-integrated multiscale correlative light and electron microscopy. J Struct Biol 201, 63-75 (2018).

11. Liu, B. et al. Three-dimensional super-resolution protein localization correlated with vitrified cellular context. Sci Rep 5, 13017 (2015).

12. Sartori, A. et al. Correlative microscopy: Bridging the gap between fluorescence light microscopy and cryo-electron tomography. Journal Of Structural Biology 160, 135-145 (2007).

13. Schorb, M. \& Briggs, J. A. Correlated cryo-fluorescence and cryo-electron microscopy with high spatial precision and improved sensitivity. Ultramicroscopy 143, 24-32 (2014).

14. van Driel, L. F., Valentijn, J. A., Valentijn, K. M., Koning, R. I. \& Koster, A. J. Tools for correlative cryo-fluorescence microscopy and cryo-electron tomography applied to whole mitochondria in human endothelial cells. Eur J Cell Biol 88, 669-684 (2009).

15. Tuijtel, M. W., Koster, A. J., Jakobs, S., Faas, F. G. A. \& Sharp, T. H. Correlative cryo super-resolution light and electron microscopy on mammalian cells using fluorescent proteins. Sci Rep 9, 1369 (2019).

16. Xu, X. et al. Ultra-stable super-resolution fluorescence cryo-microscopy for correlative light and electron cryo-microscopy. $S c i$ China Life Sci 61, 1312-1319 (2018).

17. Anderson, K. L., Page, C., Swift, M. F., Hanein, D. \& Volkmann, N. Marker-free method for accurate alignment between correlated light, cryo-light, and electron cryo-microscopy data using sample support features. J Struct Biol 201, 46-51 (2018).

18. Fukuda, Y. et al. Coordinate transformation based cryo-correlative methods for electron tomography and focused ion beam milling. Ultramicroscopy 143, 15-23 (2014).

19. Hampton, C. M. et al. Correlated fluorescence microscopy and cryo-electron tomography of virus-infected or transfected mammalian cells. Nat Protoc 12, 150-167 (2017).

20. Moser, F. et al. Cryo-SOFI enabling low-dose super-resolution correlative light and electron cryo-microscopy. Proc Natl Acad Sci USA 116, 4804-4809 (2019).

21. Schellenberger, P. et al. High-precision correlative fluorescence and electron cryo microscopy using two independent alignment markers. Ultramicroscopy 143, 41-51 (2014).

22. Schorb, M. et al. New hardware and workflows for semi-automated correlative cryo-fluorescence and cryo-electron microscopy/ tomography. J Struct Biol 197, 83-93 (2017).

23. Berriman, J. A. et al. Structural organization of Weibel-Palade bodies revealed by cryo-EM of vitrified endothelial cells. Proc Natl Acad Sci USA 106, 17407-17412 (2009).

24. Casanova, G. et al. Shrinkage of freeze-dried cryosections of cells: Investigations by EFTEM and cryo-CLEM. Micron 88, 77-83 (2016).

25. Plitzko, J. M., Rigort, A. \& Leis, A. Correlative cryo-light microscopy and cryo-electron tomography: from cellular territories to molecular landscapes. Curr Opin Biotechnol 20, 83-89 (2009).

26. Rigort, A. et al. Micromachining tools and correlative approaches for cellular cryo-electron tomography. J Struct Biol 172, 169-179 (2010).

27. Schwartz, C. L., Sarbash, V. I., Ataullakhanov, F. I., McIntosh, J. R. \& Nicastro, D. Cryo-fluorescence microscopy facilitates correlations between light and cryo-electron microscopy and reduces the rate of photobleaching. J Microsc 227, 98-109 (2007).

28. Tao, C. L. et al. Differentiation and Characterization of Excitatory and Inhibitory Synapses by Cryo-electron Tomography and Correlative Microscopy. J Neurosci 38, 1493-1510 (2018).

29. Padman, B. S., Bach, M. \& Ramm, G. An improved procedure for subcellular spatial alignment during live-cell CLEM. PLoS One 9 , e95967 (2014).

30. Bos, E. et al. Vitrification of Tokuyasu-style immuno-labelled sections for correlative cryo light microscopy and cryo electron tomography. J Struct Biol 186, 273-282 (2014).

31. Paul-Gilloteaux, P. et al. eC-CLEM: flexible multidimensional registration software for correlative microscopies. Nat Methods 14, $102-103(2017)$

32. Ou, H. D., Deerinck, T. J., Bushong, E., Ellisman, M. H. \& O’Shea, C. C. Visualizing viral protein structures in cells using genetic probes for correlated light and electron microscopy. Methods 90, 39-48 (2015).

33. Kukulski, W. et al. Correlated fluorescence and 3D electron microscopy with high sensitivity and spatial precision. J Cell Biol 192, $111-119(2011)$

34. Haring, M. T. et al. Automated sub-5 nm image registration in integrated correlative fluorescence and electron microscopy using cathodoluminescence pointers. Sci Rep 7, 43621 (2017)

35. Suloway, C. et al. Automated molecular microscopy: the new Leginon system. J Struct Biol 151, 41-60 (2005).

36. Mastronarde, D. N. Automated electron microscope tomography using robust prediction of specimen movements. J Struct Biol 152, 36-51 (2005)

37. McDonald, D. et al. Visualization of the intracellular behavior of HIV in living cells. J Cell Biol 159, 441-452 (2002).

38. Forshey, B. M., von Schwedler, U., Sundquist, W. I. \& Aiken, C. Formation of a human immunodeficiency virus type 1 core of optimal stability is crucial for viral replication. J Virol 76, 5667-5677 (2002).

39. Ganser-Pornillos, B. K., Yeager, M. \& Sundquist, W. I. The structural biology of HIV assembly. Curr Opin Struct Biol 18, 203-217 (2008).

40. Campbell, E. M., Nunez, R. \& Hope, T. J. Disruption of the actin cytoskeleton can complement the ability of Nef to enhance human immunodeficiency virus type 1 infectivity. Journal Of Virology 78, 5745-5755 (2004).

41. Kremer, J. R., Mastronarde, D. N. \& McIntosh, J. R. Computer visualization of three-dimensional image data using IMOD. J Struct Biol 116, 71-76 (1996).

42. Briggs, J. A. et al. The mechanism of HIV-1 core assembly: insights from three-dimensional reconstructions of authentic virions. Structure 14, 15-20 (2006).

43. Lee, K. et al. Flexible Use of Nuclear Import Pathways by HIV-1. Cell Host \& Microbe 7, 221-233 (2010).

\section{Acknowledgements}

We thank Dr. Teresa Brosenitsch for critical reading the manuscript. This work was supported by the National Institutes of Health P50 grant GM082251 (PZ, ZA, SCW), the UK Wellcome Trust Investigator Award 206422/Z/17/Z (PZ) and the UK Biotechnology and Biological Sciences Research Council grant BB/S003339/1 (PZ). 


\section{Author contributions}

P.Z. conceived and designed the experiments. J.N. and X.F. cultured mammalian cells. X.F. performed cryofluorescence microscopy, cryoEM/ET and correlative microscopy, developed autoCLEM workflow and associated software. Z.Z. and Z.A. produced HIV-1 fluorescence-tagged HIV-1 virus particles. S.C.W. and X.F. performed live-cell confocal fluorescence microscopy and analyzed fLM data. X.F. and P.Z. interpolated data and wrote the paper with support from other authors.

\section{Competing interests}

The authors declare no competing interests.

\section{Additional information}

Supplementary information is available for this paper at https://doi.org/10.1038/s41598-019-55766-8.

Correspondence and requests for materials should be addressed to P.Z.

Reprints and permissions information is available at www.nature.com/reprints.

Publisher's note Springer Nature remains neutral with regard to jurisdictional claims in published maps and institutional affiliations.

(c) (i) Open Access This article is licensed under a Creative Commons Attribution 4.0 International License, which permits use, sharing, adaptation, distribution and reproduction in any medium or format, as long as you give appropriate credit to the original author(s) and the source, provide a link to the Creative Commons license, and indicate if changes were made. The images or other third party material in this article are included in the article's Creative Commons license, unless indicated otherwise in a credit line to the material. If material is not included in the article's Creative Commons license and your intended use is not permitted by statutory regulation or exceeds the permitted use, you will need to obtain permission directly from the copyright holder. To view a copy of this license, visit http://creativecommons.org/licenses/by/4.0/.

(C) The Author(s) 2019 